\title{
Perforation du voile du palais suite à une intubation prolongée
}

\section{RÉSUMÉ}

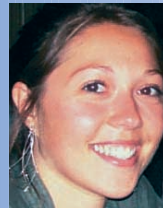

Julie GUILLET

Faculté d'odontologie de Nancy.

Julie BEMER

Faculté d'odontologie de Nancy.

Pierre BRAVETTI

Maître de conférence

Praticien hospitalier

à la faculté d'odontologie de Nancy,

Doyen de la faculté d'odontologie,

Responsable du département

de chirurgie et pathologie buccale

Service d'odontologie,

CHU de Nancy.
Un homme de 69 ans, porteur de prothèses adjointes complètes, présente une perforation au niveau du voile du palais dans un contexte d'intubation oro-trachéale prolongée. L'exploration et les examens complémentaires n'objectivent pas de pathologie tumorale. Cette perforation amène une communication bucco-pharyngée, entraînant une voix nasonnée et un reflux nasal de l'alimentation liquide.

Un obturateur vélaire en résine, solidaire de sa prothèse maxillaire, est conçu. La thérapeutique retenue est non invasive, rapide à mettre en œuvre, efficace et évolutive. Elle peut être transitoire ou constituer une alternative à la chirurgie lorsque celle-ci s'avère impossible.

AOS 2009:248:307-312 


\section{Introduction}

Les perforations palatines ont pour conséquence une communication entre les cavités orale et nasale. Elles peuvent être localisées au niveau du palais dur et/ou du palais mou. Leurs étiologies sont diverses, congénitales (fentes du massif facial) ou acquises. Ces dernières sont variées, pouvant être traumatiques, infectieuses, tumorales, consécutives à des maladies systémiques ou

\section{Cas clinique}

Monsieur X., âgé de 69 ans et édenté total, est adressé au Centre Alexis Vautrin (Centre Régional de Lutte Contre le Cancer) à Nancy suite à la découverte d'une perforation du voile du palais.

L'anamnèse révèle un cheminement complexe : le patient, présentant un rétrécissement aortique calcifié serré, a été hospitalisé dans le Département de chirurgie cardiovasculaire et transplantation du CHU de Nancy afin de subir un remplacement valvulaire aortique par prothèse mécanique. Les antécédents de ce patient sont lourds : hypertension artérielle, insuffisance rénale dialysée, dyslipidémie, multiples antécédents d'angioplastie (membres inférieurs, artère interventriculaire, carotide).

L'intervention de chirurgie cardiaque s'est bien déroulée et les suites immédiates sont simples. Cependant, une hypoxémie importante à $J 6$ oblige la réintubation du patient, qui est ensuite transféré en réanimation médicale où il restera intubé pendant un mois.

Sur le plan ORL, on note après dix jours d'intubation l'apparition d'une épistaxis bilatérale encore à une consommation abusive de cocaïne par voie nasale [1, 2]. Cependant, seule une anamnèse minutieuse et les examens clinique et anatomopathologique permettent la détermination de l'étiologie.

La perforation palatine reste une entité rare dont le traitement, en l'absence de fermeture spontanée, peut être chirurgical ou prothétique [3].

prédominant à gauche, motivant la transfusion d'un culot globulaire et d'un concentré plaquettaire. Lors de la consultation ORL le jour même, un méchage est réalisé sur un saignement actif et pulsatile venant du cavum et sans possibilité de coagulation locale. On note après l'extubation l'apparition de fausses routes, contre-indiquant temporairement l'alimentation per os. Une sonde nasogastrique est alors mise en place mais, dès son retrait, le patient se plaint de reflux liquidiens, sa voix est nasonnée et il est revu en consultation ORL.

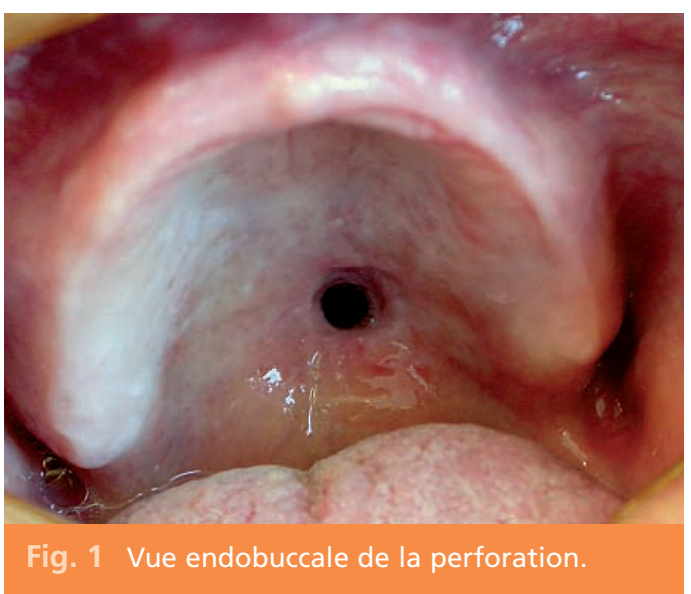


Lors de l'examen clinique, une perforation du voile du palais, d'environ $7 \mathrm{~mm}$ de diamètre et latéralisée à gauche est constatée (fig. 1). Des biopsies de principe sont réalisées afin d'éliminer une lésion cancéreuse. Les examens anatomopathologiques ne décèlent ni dysplasie ni structure carcinomateuse.
Le patient est alors adressé à l'unité d'odontologie du Centre Alexis Vautrin où une réhabilitation prothétique est réalisée. En effet, les berges de la perforation étant épithélialisées, une fermeture spontanée est improbable. Par ailleurs, au vu de l'état général du patient et de ses antécédents, une fermeture chirurgicale n'est pas envisageable.

\section{Prise en charge prothétique}

Monsieur X. est porteur de prothèses complètes bimaxillaires qui, bien qu'anciennes, sont bien adaptées et tolérées. Le traitement de choix est donc de réaliser un prolongement en résine partant du bord postérieur de sa prothèse maxillaire, afin de venir obturer la perforation.

Une empreinte fonctionnelle de la perforation est effectuée, en utilisant la prothèse du patient, et ceci en deux temps : tout d'abord à la pâte de Kerr afin de confectionner un tuteur rigide, puis celui-ci est enduit de silicone light pour obtenir une empreinte de précision (fig. 2 a). Un silicone lourd (de type Putty) est ensuite placé dans l'intrados prothétique, et

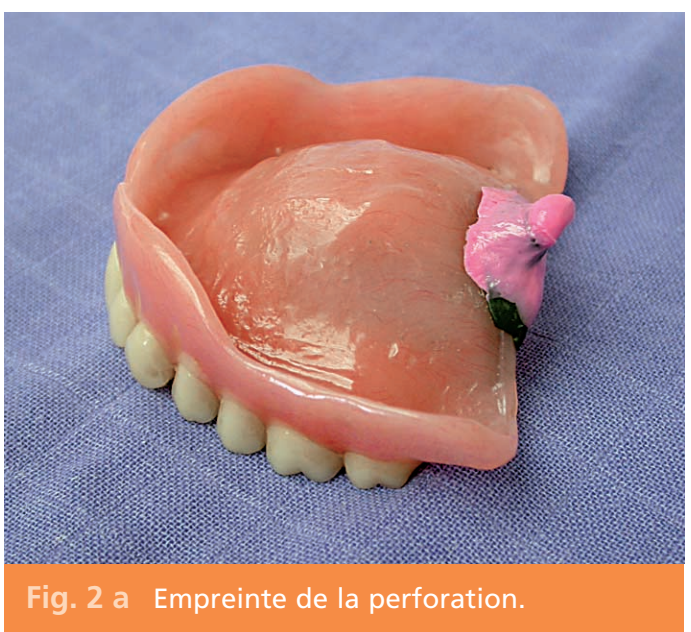

l'ensemble est isolé avant d'être coulé en plâtre (fig. 2 b).

Une fois l'empreinte démoulée, la prothèse est ébouillantée afin de retirer totalement la pâte de Kerr résiduelle. Une résine autopolymérisable est ensuite mise en place dans le modèle en plâtre obtenu et la prothèse y est repositionnée, de façon précise et aisée grâce au silicone lourd. L'ensemble est mis sous pression dans une cocotte pendant 5 minutes. Le modèle en plâtre est alors cassé pour démouler la prothèse sans risquer de fracturer le prolongement en résine visant à obturer la perforation (fig. 3 a et $\mathbf{3}$ b).

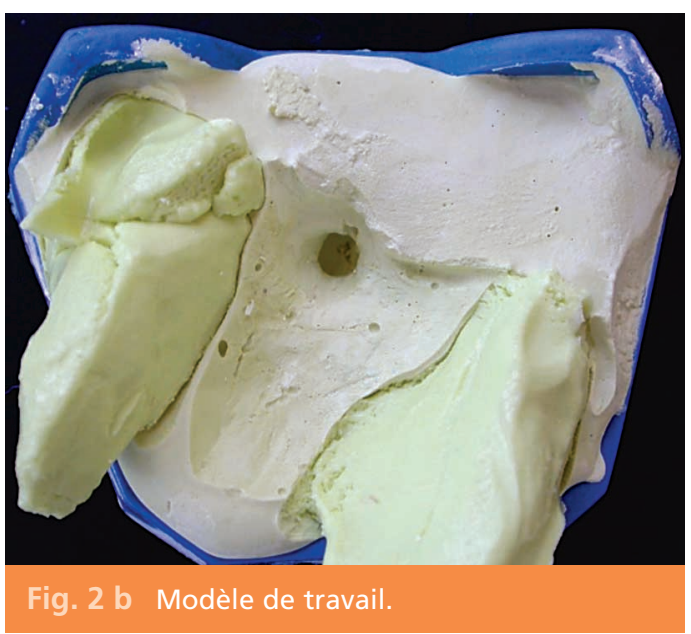



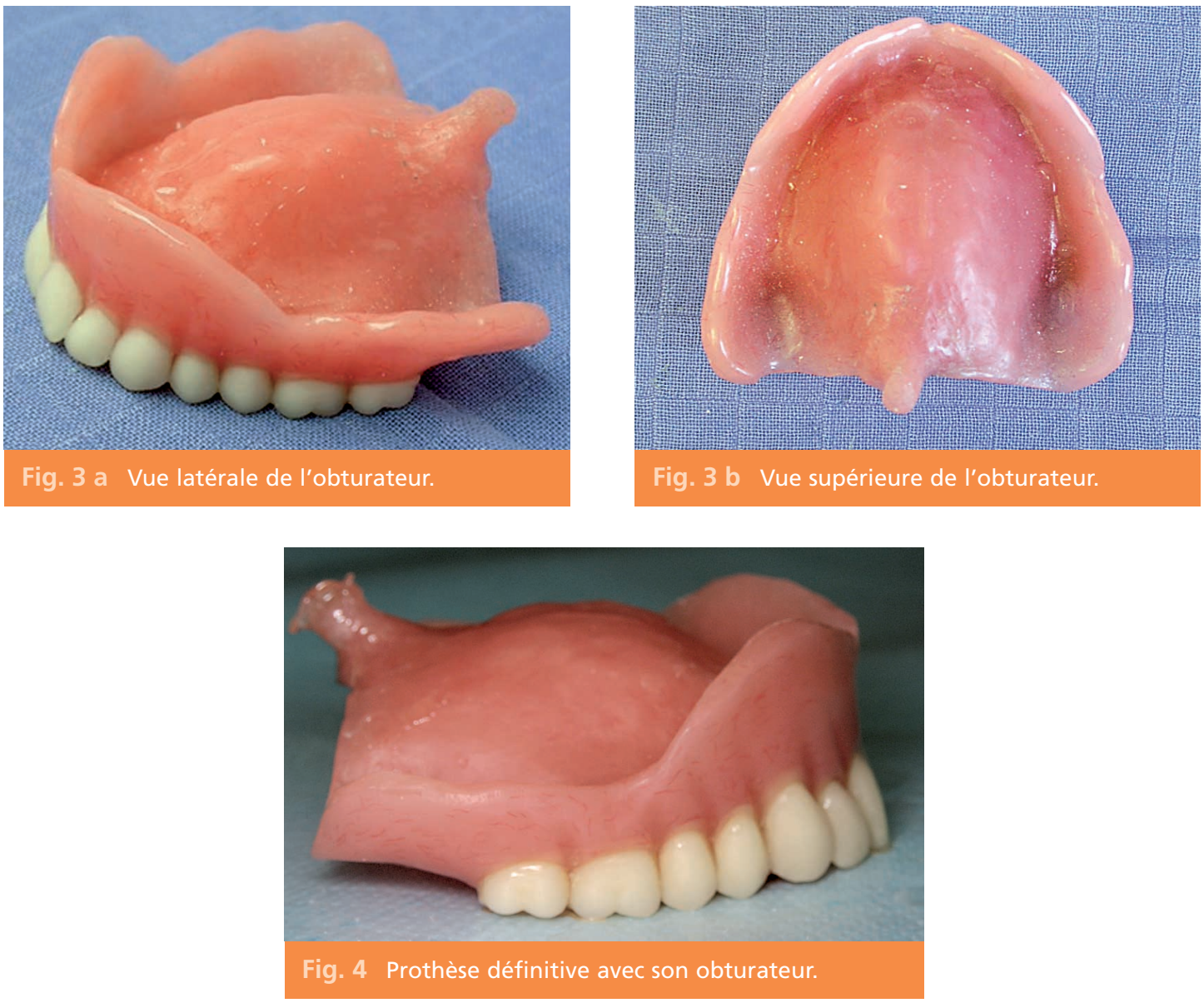

L'essayage de la prothèse est réalisé dans la même séance et son extension postérieure est tolérée. On constate immédiatement la disparition du nasonnement de la voix. En faisant boire un verre d'eau au patient, on note également la suppression du reflux liquidien par le nez. L'étanchéité est encore améliorée par la mise en place d'un silicone light de type Dropstill sur le prolongement en résine.

\section{Discussion}

Dans le cas présenté ici, l'étiologie la plus probable de la perforation constatée est une lésion de compression, de type escarre, due à une intubation prolongée et iatrogène.
Dans un second temps, de nouvelles prothèses ont été confectionnées (fig. 4). Les empreintes primaires à l'alginate ont été réalisées de façon classique, mais tout en englobant la perforation. Par la suite, le prolongement en résine a été conçu de la même façon que sur la prothèse initiale du patient.

La perforation palatine, notamment du palais mou, reste une entité rare en dehors du contexte de la chirurgie carcinologique. Les étiologies sont variables et les lésions chro- 
niques liées à une intubation oro-trachéale sont exceptionnelles. En effet, davantage de traumatismes aigus occasionnés lors du passage de la sonde d'intubation sont observés $[4,5]$. Seul un cas de perforation palatine consécutive à une intubation prolongée a été rapporté dans la littérature à ce jour [6].

La prise en charge dépend à la fois de l'étiologie et du diamètre de la perforation. Lorsque celle-ci est inférieure à $10 \mathrm{~mm}$, une fermeture spontanée peut survenir, sauf si les berges sont déjà épithélialisées. Lorsque le diamètre est supérieur à $10 \mathrm{~mm}$, une fermeture chirurgicale par lambeaux est généralement envisagée [3]. Dans les deux cas, la réussite du traite-

\section{Conclusion}

Ce cas souligne l'importance de la surveillance des patients restant longtemps intubés. II est nécessaire de vérifier la position de la sonde d'intubation, de façon à ce que celle-ci soit placée de façon non compressive sur les tissus exoet endobuccaux. Un changement régulier de côté de la sonde peut également être envisagé, pour limiter la durée des appuis sur une même zone. ment est bien sûr conditionnée par la suppression de l'étiologie, notamment chez les toxicomanes ou lors d'un traumatisme.

Dans le cas présent, seule une réhabilitation prothétique était possible, le patient étant trop affaibli pour supporter une autre intervention chirurgicale dans l'immédiat. Une surveillance semestrielle est préconisée, avec remplacement du silicone light sur la partie obturatrice en cas de réapparition du reflux liquidien.

Ce type de traitement prothétique peut tout à fait être une solution transitoire, en attendant une amélioration de l'état général du patient qui permettrait alors une fermeture chirurgicale.

Bien que ce cas soit exceptionnel de par son étiologie, la perforation du voile du palais est un véritable handicap fonctionnel pour les patients. Leur prise en charge correspond en priorité à une amélioration de leur qualité de vie, et une approche multidisciplinaire peut permettre de trouver des alternatives à la chirurgie lorsque celle-ci n'est pas envisageable.

\section{Bibliographie}

1. Von Arx DP, Cash AC. Spontaneous palatal fenestration:

review

of the literature

and report

of a case.

$\mathrm{Br} J$ Oral Maxillofac Surg 2000;38(3):235-7.
2. Di Cosola M et al. Cocaine-related syndrome and palatal reconstruction: report of a series of cases. Int J Oral Maxillofac Surg 2007;36(8):721-7.
3. Karabulut $A B$ et al. Hard palate perforation due to invasive aspergillosis in a patient with acute lymphoblastic leukemia. Int J Pediatr Otorhinolaryngol 2005;69(10):1395-8 
4. Atkinson CJ, Rangasami J. Uvula necrosis an unusual cause of severe postoperative sore throat.

$\mathrm{Br} J$ Anaesth 2006;97(3):426-7.
5. Vincent RD Jr et al. Soft palate perforation during orotracheal intubation facilitated by the GlideScope videolaryngoscope. J Clin Anesth 2007;19(8):619-21.
6. Ozgul S,

Tezel E,

Numanoglu A.

Palatal perforation

after a long intubation

period.

Eur J Plast Surg

2005;27:335-7.

\section{SUMMARY}

\section{Soft palate perforation following a long during intubation}

Julie GUILLET,

Julie BEMER

\section{Keywords \\ - oral fistula \\ - soft palate \\ - palatal obturator \\ - soft tissue injuries \\ intratracheal \\ (endotracheal) \\ intubation}

A 69 years old man wearing complete dentures presents a soft palate perforation following a long-during intubation. Complementary investigations do not reveal any tumoral process. This perforation leads to an oropharyngeal communication, with dysphonia and nasal reflux of liquid aliments.

A resin soft palate obturator, fixed to the existing prosthesis, is realized. This therapeutic is a non-invasive, quick, efficient and evolutive method. It may be an alternative to surgery when this one is impossible. 